\title{
Editorial
}

\section{Integration of Medical Images into the Digital Hospital}

\author{
Xiaohong Gao ${ }^{*}, 1$, , Henning Müller ${ }^{2, \dagger}$ and Thomas M. Deserno ${ }^{3, \dagger}$ \\ ${ }^{I}$ School of Engineering and Information Sciences, Middlesex University, London NW4 4BT, UK \\ ${ }^{2}$ Business Information Systems, University of Applied Sciences, Western Switzerland (HES-SO), TechnoArk 3, 3960 \\ Sierre, Switzerland \\ ${ }^{3}$ Department of Medical Informatics, RWTH Aachen University, Pauwelsstr. 30, D-52057 Aachen, Germany \\ $\S_{\text {Guest Editor }}$ \\ ${ }^{\dagger}$ Co-Guest Editor
}

Medical imaging has revolutionised healthcare delivery and become a hallmark of modern medicine in the last 30 years, benefiting the world to a large extent. This development coupled with the advances of information and communications technology (ICT) has enabled the delivery of high quality healthcare that had not been able to achieve before. On the other hand, the world has moved on beyond the current reactive model of 'one-size-fits-all' and hamstrung by the problems of fragmentation and lack of coordination of distributed data. Medical images are of a typical example. While picture archiving and communications systems (PACS) have come closely to be a universal tool in managing medical images, they only work for radiology images and are searchable by using text descriptions. With the rapidly growing of image volumes, obtaining a relevant datum using only key words is like finding a needle in a haystack. Additionally, with each clinical centre specialising in different domains, medical images collected are fragmented and of many forms. A novel data retrieval architecture should ensure queries not only being able to span to data sources of distributed collections, but also without prior standardisation of the original data, entailing each database with scope, scale, and the necessary flexibility with interoperability, i.e., the databases established today will be still in operation tomorrow in the rapidly progressing digital era

With this in mind, this special issue has made a concerted effort to explore the breadth of medical imaging innovations and constitutes a suite of papers from a number of leading researchers in these fields, covering a wide range of topics that include novel imaging tools, robust image analysis software, and future sustainable data management systems, with a focus on image interpretation, IT infrastructure and database integration respectively.

*Address correspondence to this author at the School of Engineering and Information Sciences, Middlesex University, London NW4 4BT, UK; Tel: +44 (0) 208411 Fax: +44 (0) 208411 2252;

E-mail: x.gao@mdx.ac.uk
This issue begins with the paper on the introduction of a new, non-invasive imaging technique, phase contrast imaging (PCI), by Zhang and Luo (Paper 1). Built on X-ray phase shift, PCI has the ability of revealing soft tissues. Therefore, it has been applied in the establishment of models of hepatic fibrosis in a mouse liver, construction of a threedimensional morphology of a segment of blood vessels, and imaging the alveoli in a lung, paving the way on diagnosis of respiratory disease in a non-invasive way in the future.

In terms of interpretation of images, Paper 2, written by $\mathrm{Su}$, Wang, Jiao, and Guo, has developed a novel computer aided diagnosis (CAD) system in a fully automatic manner to detect and classify breast tumours based on ultrasonic images, in assisting clinicians in arriving at a correct diagnosis, especially in distinguishing benign from malignant tumours. In addition, in order to recover vital information interlaced between frames in a series of 3D ultrasonic images, Lin and $\mathrm{Li}$ have developed a novel approach in their 4D echocardiography system as detailed in Paper 3. By which, they introduce the method of omnidirectional M-mode to the data that are lost while analysing images on a still 2D basis. In this way, the motion parameters related to the heart can be calculated precisely.

In addition, imaging techniques have made a huge impact on modern medicine and are employed in a number of cutting edge applications. Paper 4 demonstrates a clinical application in the field of image-guided robotic vascular intervention based on angiography. Written by surgeons of Tian, Jia, Lu, and Hui, the paper starts with the introduction of a robotic system that is employed to repair damaged vascular vessels. Then, experiments have been conducted to evaluate the accuracy of the system and future directions are given.

Significantly, with such huge amount of images acquired everyday, systematic management of these data remains to be paramount. At present, all the images in a clinical environment are retrievable by text. As much as we enjoy the use of text-based search for relevant data, searching an image database based on an sample image is also welcome, especially when a certain amount of information is not 
expressible in words. To this end, the ImageCLEF benchmark was established in 2003 to facilitate this initiative and has seen participations by an increasing number of researchers. Paper 5 authored by Gkoufas, Morou, and Kalamboukis, has addressed the two-year-experience in taking part in the ImageCLEF evaluation task and presented their findings on the investigation of fusion between visual and semantic sources of images with employment of an ontology.

To extend the discussion, especially to cement the gap between low level image features and high level semantic, Paper 6 stresses the issue of content-based image retrieval (CBIR) in clinical applications. Although still in a research realm, four domain-specific CAD systems have been identified with a high degree of "user readiness". Prototype applications exist that could be delivered to clinical practice in the near future. The authors, Depeursinge, Fischer, Müller, and Deserno, also point to future directions of CBIRbased CAD systems. In particular, a number of guidelines have been proposed towards data integration and system infrastructure.

As a closing remark, Gao, Qian, and Hui have strengthened the findings by the authors from the above papers in the final paper of this issue (Paper 7), and given a summative account on the journey of modern medicine beginning from the creation of medical imaging technologies, to the establishment of image repositories, and leading to the directions of potential innovations that can be created from the existing rich collections of enormous quantities of image date. The novelty of the paper is on the building of a 'fit for purpose' online e-repository, the Middlesex medical Image Repository with a CBIR ArchivinG Environment (MIRAGE, http://image.mdx.ac.uk/ mirage), while facilitating 3D medical images with a CBIR retrieval tool and annotating images in a fully automatic way, leading to an infrastructure of allowing local development of light weight, incremental and flexible databases.

It is anticipated that this special issue throws a light on the latest development on imaging techniques, image interpretation, archive and retrieval, and clinical applications of image repositories, at the beginning of the decade, and offers the research directions in the field, aiming at the arrival of the ultimate digital hospital (paper-less) in the future.

(C) Gao et al.; Licensee Bentham Open.

This is an open access article licensed under the terms of the Creative Commons Attribution Non-Commercial License (http://creativecommons.org/licenses/by-nc /3.0/) which permits unrestricted, non-commercial use, distribution and reproduction in any medium, provided the work is properly cited. 